\title{
Best time to wait for the improvement of the sperm parameter after varicocelectomy: 3 or $\mathbf{6}$ months?
}

\author{
Mohammad Ali Ghaed ${ }^{1}$, Seyed Alireza Makkian ${ }^{2}$, Asaad Moradi ${ }^{1}$, Robab Maghsoudi ${ }^{1}$, \\ Alireza Gandomi-Mohammadabadi ${ }^{3}$ \\ ${ }^{1}$ Urology Department, Firoozgar Hospital, Iran University of Medical Sciences, Tehran, Iran; \\ 2 Iran University of Medical Sciences, Tehran, Iran; \\ ${ }^{3}$ Student Research Committee, School of Medicine, Iran University of Medical Sciences, Tehran, Iran.
}

\begin{abstract}
Summary Objective: To estimate the duration of time required following varicocelectomy to wait for the improvements of semen parameters. Therefore, we characterized the changes with the time in sperm parameters in men after varicocelectomy.

Materials and methods: In this prospective cohort study we included consecutively observed men who underwent varicocelectomy between September 2017 and September 2018 in a referral academic hospital. Clinical data of the patients, as well as their semen parameters, were measured before surgery and at 3 and 6 months afterward.

Results: In this study, a total of 100 men with average age of $29.5 \pm 6.2$ years were included. Mean sperm concentration and sperm motility significantly improved by $3(p<0.05)$, but not by 6 months following varicocelectomy. The semen volume and sperm with normal morphology were the same before and after surgery $(p>0.05)$. There was no statistically significant difference in the improvement of semen parameters when comparing 6 months to 3 months postoperatively $(p>0.05)$.

Conclusions: Sperm parameters (concentration and motility) improve by 3 months after varicocelectomy without further improvements. Consequently, physicians should decide quickly after 3-month of varicocelectomy if surgery has been not helpful and then plan other therapies, like assisted reproductive technology (ART) for managing infertility in couples.
\end{abstract}

KEY WORDS: Varicocelectomy; Male infertility; Semen parameters; Time factors; ART.

Submitted 2 March 2020; Accepted 15 March 2020

\begin{abstract}
INTRODUCTION
Varicocele is the most common but correctable cause of infertility in men and is defined as dilated tortuous veins of the pampiniform plexus in the spermatic cord (1).

Its prevalence rate is $30 \%$ in men with primary infertility, up to $80 \%$ in men with secondary infertility and 15\% in general adult male population (2). The pathophysiology of varicocele-associated infertility has not been well defined. It is thought to be related to the impairment of spermatogenesis induced by increased testicular blood flow, scrotal hyperthermia, reflux of renal and adrenal metabolites, hypoxia from venous stasis and endocrine dysfunction $(3,4)$.

In current practice, varicocelectomy, drug therapy and Assisted Reproductive Technology (ART) including intrauterine insemination (IUI), in vitro fertilization (IVF), or intracy-
\end{abstract}

toplasmic sperm injection (ICSI) are some modalities approved for men with varicocele-associated infertility (2). To date, varicocelectomy is one of the most popular modality. There are various ways to perform varicocele repair, including surgical ligation (with or without microscopic approach), laparoscopic surgery and embolization (5-8).

The effectiveness of varicocelectomy on male fertility has remained in doubt $(3,9)$. Numerous studies including systematic reviews on clinical results of varicocelectomy have reported improvements in fertility rates and semen parameters (10-14). However, some studies have reported controversial results, consisting of an unstable improvement in fertility following surgery or even worsened semen parameter (15-17).

For both couples and physicians, it is important to know the time needed to wait after varicocelectomy for the best improvement in semen parameters. So they can assess better the changes in semen parameters and the need to plan on other therapies as invasive forms of ART (18). According to the report of the Practice Committee of American Society for Reproductive Medicine in 2014, time to improvement in semen parameters following varicocelectomy is usually 3 to 6 months (19). While this has become a common practice, there are only a few studies that assess the time needed to wait for the improvement of sperm parameters following varicocelectomy.

Therefore, this study was evaluated the time-dependent changes in semen parameters in men who underwent microsurgical inguinal varicocelectomy and had semen analyses at baseline, and 3 and 6 months following surgery to estimate the duration of time required to wait for improvement of semen parameters.

\section{Materials and MEthods}

This prospective cohort study was conducted in consecutive men who underwent unilateral inguinal varicocelectomy. It was carried out in a referral academic hospital between September 2017 and September 2018 after approval of ethical committee of Iran University of Medical Sciences. All patients filled an informed written consent form. Inclusion criteria were infertile men who had a leftsided palpable varicocele (grade I/II/III) and were further documented with Doppler duplex ultrasound with at least

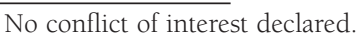


one or more abnormal semen parameter and also adolescents who had left palpable varicocele and objective evidence of reduced ipsilateral testicular size more than 20\% is in comparison with the right testis (19). Infertility was defined as male partner who was unsuccessful to achieve successful fertility at least after 12 months of regular unprotected sexual intercourse or couples who had been able to get pregnant at least once, but now is unable with female partners normal in fertility re gynecologist.

The semen samples from infertile individuals were classified according to the WHO-2010, and samples with a sperm concentration $<15 \mathrm{million} / \mathrm{ml}$ (oligozoospermic) and/or progressive motility $(a+b)<32 \%$ (asthenozoospermic) and/or morphologically normal sperm $<4 \%$ ( teratozoospermic) considered as abnormal.

The largest vein diameter and reversal blood flow of pampiniform plexus of veins were measured. Diagnosis of clinical varicocele was based on 2 criteria: 1) Left-sided venus diameter cut-point values of $2.5 \mathrm{~mm}$ in rest or $3 \mathrm{~mm}$ during Valsalva and 2) presence of retrograde flow (20).

Exclusion criteria were additional causes of infertility, significant medical diseases, leucocytospermia, azoospermia, patients with a history of probable sexually transmitted diseases and previous history of scrotal or inguinal trauma or surgery.

All patients' information including demographic information (age, marriage, number of children) and smoking habits were obtained and entered into the checklist. Then patients underwent microsurgical inguinal varicocelectomy by a single expert urologist.

Masturbation was used to collect semen samples following 3-day period of sexual abstinence. The samples were analyzed within 1 hour after collection to measure the ejaculation volume, sperm concentration, motility and morphology. To eliminate the effects of different test kits from different laboratories, three-stage trials of patients were conducted in a single laboratory. Based on the last guidelines of the World Health Organization (WHO) (2010), computer-aided sperm analysis (CASA) was used to assess sperm concentration, motility and morphology. We documented any pregnancy that might occur during the study period. Semen analyses were obtained before, 3 and 6 months after surgery. We followed the participants for 6 months after the day of surgery.

The primary outcome measure was characterizing the time-dependent changes of each semen parameter during. Secondary outcomes were determining spontaneous pregnancy rate after intervention during 6 months follow-up. The provided data were analyzed by SPSS 18 (statistical package for social sciences, Chicago, IL). Continuous variables with normal distribution were described using

Table 2.

Changes in semen parameters at 3 and 6 months after surgery.

\begin{tabular}{|c|c|c|c|c|c|c|}
\hline \multirow[b]{2}{*}{ Parameters* } & \multicolumn{3}{|c|}{ Timing of semen analysis according to surgery } & \multicolumn{3}{|c|}{ P-Value } \\
\hline & Preoperative & 3 Months & 6 Months & Before vs. 3 Mo & Before vs. 6 Mo & 3 Mo After vs. 6 Mo \\
\hline Semen volume (mL) & $2.91 \pm 1.36$ & $2.94 \pm 1.22$ & $2.93 \pm 1.30$ & .272 & .741 & .852 \\
\hline Sperm concentration (million/mL) & $16.33 \pm 20.41$ & $19.36 \pm 20.66$ & $17.79 \pm 15.36$ & .012 & .120 & .903 \\
\hline Sperm morphology (\% normal): & $50.01 \pm 21.51$ & $51.40 \pm 19.57$ & $49.79 \pm 18.80$ & .229 & .657 & .192 \\
\hline Progressive motile sperm count (\%) & $42.43 \pm 22.04$ & $47.98 \pm 19.01$ & $46.48 \pm 21.58$ & .001 & .075 & .194 \\
\hline
\end{tabular}

means \pm SEM (standard error of mean). Paired t-test was used for comparison between the pre- and post-surgery. Wilcoxon signed-ranks test was used for the parameters with a non-normal distribution. P-value less than 0.05 were considered statistically significant.

\section{Results}

One-hundred patients (mean age $=29.5 \pm 6.2$ years; range: $17-46$ ) with various grades of varicocele were enrolled in our study. All patients had a left-sided varicocele; six patients (6\%) had grade 1 varicocele, 18 (18\%) had grade 2 , and $76(76 \%)$ had grade 3 . Among our patients, 65 patients were married: 39 of them had primary infertility and 26 of them had secondary infertility. The mean of vein dilatation was $3.62 \pm 1.2$ and the mean of reflux duration in pampiniform plexus of veins was $2.3 \pm 1.8$ seconds.

Sperm concentration among 78 patients was under 15 million/ml, progressive motility among 49 patients was under 32\% and normal morphology among 7 patients was under 4\%. Therefore, the 100 individuals with grades I, II \& III varicocele were sub-grouped to oligoasthenoteratozoospermic $(n=2)$, oligoteratozoospermic $(n=5)$, oligoasthenozoospermic $(n=25)$ oligozoospermic $(n=46)$ and asthenozoospermic $(n=22)$. Other demographic data are listed in Table 1.

Sperm concentration and motilities were significantly increased by 3 months but not by 6 months following surgical therapy compared with preoperative. No further significant improvement was found in sperm concentration or motility after 3 months up to 6 months after surgery. Semen volume and the proportion of sperm with normal morphology did not significantly improve during the study. In Table 2 we summarized the findings of semen parameters analysis before, 3 and 6 months after varicocelectomy. Also 24 of the 65 married patients' wives (37\%)

\section{Table 1.}

Demographic and baseline characteristics of participants.

\begin{tabular}{|lc|}
\hline Characteristic & Patients $(\mathbf{n}=\mathbf{1 0 0})$ \\
\hline Age $(\mathrm{Y})$ & $29.5 \pm 6.2$ \\
\hline Marriage, $\mathrm{n}(\%)$ & $65(65 \%)$ \\
Married & $35(35 \%)$ \\
Single & $67(67 \%)$ \\
\hline Smoking, $n(\%)$ & $39(39 \%)$ \\
\hline Type of infertility, $n(\%)$ & $26(26 \%)$ \\
Primary & $6(6 \%)$ \\
Secondary & $18(18 \%)$ \\
\hline Grade of varicocele, $n(\%)$ & $76(76 \%)$ \\
1 & \\
2 & \\
3 & \\
\hline
\end{tabular}


got pregnant, including 19 (49\%) and 5 (19\%) in patients with primary and secondary infertility, respectively.

\section{Discussion}

The most common diagnosed cause of infertility in men is varicocele, and about $30 \%$ of infertile men with primary infertility have varicocele. Many factors such as altered venous pressure, hyperthermia, testicular blood flow, oxidative stress, hormonal imbalance, toxic substances, genetic factors, and lifestyle are among factors that are supposed to have a role in varicocele (21).

In this study, our patients underwent microsurgical varicocelectomy, which has been regarded as the gold standard in comparison with other surgical techniques and radiological management such as retrograde embolization in terms of the recurrence rate and the pregnancy rate $(22,23)$. While there are conflicting findings regarding the effects of varicocelectomy on fertility rates in couples with infertility, strong accepted evidence and reports suggest it has a positive effect on male reproduction because it upgrades semen parameters, reduces sperm DNA fragmentation, improves ART outcomes and increases spontaneous pregnancy rates (24).

Two meta-analysis by Agarwal et al. (3) and Marmar et al. (25) in 2007 assessed the effects of varicocelectomy on semen parameters, and concluded that surgical varicocelectomy has a positive effect on all semen parameters in infertile men with a clinical varicocele. In this study, we reported significant increase in sperm concentration and motility at 3 months but not at 6 months after varicocelectomy. However, we did not observe significant improvement in other parameters including sperm morphology and semen volume.

Revised WHO manual (2010) has recommended CASA system as a selective means to analyze semen samples, that it has not been routinely used before 2010 for semen analysis. In this study, we performed semen analysis by using this new method based on the guidelines of the WHO (2010) and confirmed the varicocelectomy effect by a more accurate system. Ariagno et al. (26) also used CASA to assess semen parameters. However, improvement of all semen parameter was insignificant in contrast to our study. This difference in the improvement of sperm parameters between different studies can be related to the type of male factor infertility, heterogeneity of intrasamples, type of surgery, sample sizes, type of semen assessment, etc.

In addition, varicocelectomy, a low-risk modality with minimal morbidity, has many advantages over the simple improvement of sperm parameters. It could improve other parameters of sperm quality that we do not assess in standard semen testing, such as sperm deoxyribonucleic acid fragmentation rates. Alargkof V, et al. (27), Zini et al. (28) and Smit et al. (29) found that there is a higher degree of DNA fragmentation in patients with varicocele and significantly improvements after varicocelectomy of semen parameters and DNA fragmentation rates in men with infertility.

Previously studies demonstrated varicocelectomy potentially improves semen parameters that lead to applying less invasive and expensive forms of ART or even natural pregnancy (30). In past years, several options other than varicocelectomy such as IUI and IVF have been introduced to improve couples' chance of conceiving (31). Accordingly, it has become a common concern for couples to know the duration needed to wait for the improvement of semen parameters after varicocelectomy; since it is unacceptable for most couples, especially those with an elderly woman, to wait a long time for the outcome of varicocelectomy to become clear. Instead, they prefer to continue directly with IUI or IVF, resulting in missing the benefit of varicocelectomy.

Regarding these findings, in this study we retrospectively investigated the time-dependent changes in the semen parameters results in 100 men following varicocelectomy to estimate the time needed to note improvements in semen parameters. Improvements in the semen parameters after 3 months following surgery were not significant in our patients. Al Bakri et al. (32) and Fukuda et al. (24) reported similar findings to our study.

These findings suggest that up to 3 months after surgery, improvement in semen parameters could occur, and further improvement after that time in unexpected. Therefore, varicocelectomy is an acceptable option for men with clinical varicocele, even for couples with advanced female age; because they could quickly proceed to ART if their semen parameters did not improve up to 3 months after the surgery.

In this study spontaneous pregnancy was achieved in $37 \%$ of couples during a 6 -month period after varicocelectomy, and rate of pregnancy was higher in patients with primary infertility (49\%) in comparison with secondary infertility (19\%). Abdel-Meguid TA et al. (9) reported a pregnancy rate of $36.4 \%$ during a 12 -month follow-up in patients who underwent surgery and concluded varicocelectomy increase odds of spontaneous pregnancy within 1 year of follow-up which is consistent with our results. Contrary to these findings, Nieschlag et al. (33), found no difference in the odds of pregnancy in varicocele-treated patients compared with no treatment, suggesting no benefit for varicocele treatment

There are several limitations to this study. Initially, it had a relatively small sample size and was a non-randomized study without a control group. Therefore, the findings of this study need to be confirmed with a larger group to determine definite results. In addition, we followed our patients up to 6 months after surgery. As pregnancy is the ultimate goal for infertility due to this short duration of observation in patients, for efficient evaluation of pregnancy rate, we should consider at least 1-year follow-up, and compare results of intervention group with a control group. Finally, it is better to investigate the characteristics data of the female partner, as her age because of its impact on the decision-making process in couples.

\section{Conclusions}

Sperm parameters (concentration and motility) improve by 3 months following varicocelectomy and then do not improve further by 6 months. This finding could help couples and physicians evaluate better the outcome of surgery and then if required, plan on the use of other approaches, such as ART to manage infertility without prolonged delay. 


\section{ACKNOWLEDGMENT}

We would like to show our gratitude to the Rasoul Akram Hospital Clinical Research Development Center (RCRDC) for its technical and editorial assists.

\section{REFERENCES}

1. Chen YW, Niu YH, Wang DQ, et al. Effect of adjuvant drug therapy after varicocelectomy on fertility outcome in males with varicocele-associated infertility: Systematic review and meta-analysis. Andrologia. 2018; 50:e13070.

2. Vahidi S, Moein M, Nabi A, Narimani N. Effects of microsurgical varicocelectomy on semen analysis and sperm function tests in patients with different grades of varicocele: Role of sperm functional tests in evaluation of treatments outcome. Andrologia. 2018; 50:e13069.

3. Agarwal A, Deepinder F, Cocuzza M, et al. Efficacy of varicocelectomy in improving semen parameters: new meta-analytical approach. Urology. 2007; 70:532-8.

4. Baigorri BF, Dixon RG. Varicocele: A Review. Semin Intervent Radiol. 2016; 33:170-6.

5. Cayan S, Shavakhabov S, Kadioglu A. Treatment of palpable varicocele in infertile men: a meta-analysis to define the best technique. J Androl. 2009; 30:33-40.

6. Williams DH, Karpman E, Lipshultz LI. Varicocele: surgical techniques in 2005. Can J Urol. 2006; 13 Suppl 1:13-7.

7. Al-Kandari AM, Shabaan H, Ibrahim HM, et al. Comparison of outcomes of different varicocelectomy techniques: open inguinal, laparoscopic, and subinguinal microscopic varicocelectomy: a randomized clinical trial. Urology. 2007; 69:417-20.

8. Al-Said S, Al-Naimi A, Al-Ansari A, et al. Varicocelectomy for male infertility: a comparative study of open, laparoscopic and microsurgical approaches. J Urol. 2008; 180:266-70.

9. Abdel-Meguid TA, Al-Sayyad A, Tayib A, Farsi HM. Does varicocele repair improve male infertility? An evidence-based perspective from a randomized, controlled trial. Eur Urol. 2011; 59:455-61.

10. Ghaed MA, Mahmoodi F, Alizadeh HR. Prognostic factors associated with bilateral, microsurgical vasovasostomy success. Middle East Fertility Society Journal. 2018; 23:373-6.

11. Goldstein M, Gilbert BR, Dicker AP, et al. Microsurgical inguinal varicocelectomy with delivery of the testis: an artery and lymphatic sparing technique. J Urol. 1992; 148:1808-11.

12. Kibar Y, Seckin B, Erduran D. The effects of subinguinal varicocelectomy on Kruger morphology and semen parameters. J Urol. 2002; 168:1071-4

13. Marmar JL, Kim Y. Subinguinal microsurgical varicocelectomy: a technical critique and statistical analysis of semen and pregnancy data. J Urol. 1994; 152:1127-32.

14. Segenreich E, Shmuely $H$, Singer $R$, Servadio C. Andrological parameters in patients with varicocele and fertility disorders treated by high ligation of the left spermatic vein. Int J Fertil. 1986; 31:200-3.

15. Breznik R, Vlaisavljevic V, Borko E. Treatment of varicocele and male fertility. Arch Androl. 1993;30:157-60.

16. Krause W, Muller HH, Schafer H, Weidner W. Does treatment of varicocele improve male fertility? results of the 'Deutsche Varikozelenstudie', a multicentre study of 14 collaborating centres. Andrologia. 2002; 34:164-71.

17. Rageth JC, Unger C, DaRugna D, et al. Long-term results of varicocelectomy. Urol Int. 1992; 48:327-31.

18. Masterson TA, Greer AB, Ramasamy R. Time to improvement in semen parameters after microsurgical varicocelectomy in men with severe oligospermia. Can Urol Assoc J. 2019; 13:E66-E9.

19. Practice Committee of the American Society for Reproductive Medicine; Society for Male Reproduction and Urology. Report on varicocele and infertility: a committee opinion. Fertil Steril. 2014; 102:1556-60.

20. Pilatz A, Altinkilic B, Kohler E, et al. Color Doppler ultrasound imaging in varicoceles: is the venous diameter sufficient for predicting clinical and subclinical varicocele? World J Urol. 2011; 29:645-50.

21. Tavalaee M, Bahreinian M, Barekat F, et al. Effect of varicocelectomy on sperm functional characteristics and DNA methylation. Andrologia. 2015; 47:904-9.

22. Diegidio P, Jhaveri JK, Ghannam S, et al. Review of current varicocelectomy techniques and their outcomes. BJU international. 2011; 108:1157-72.

23. Ughi G, Dell'Atti L, Ricci C, Daniele GP. (Micro)surgical and percutaneous procedures in the management of varicocele: 25 years of experience. Arch Ital Urol Androl 2012; 84:79-83.

24. Fukuda T, Miyake H, Enatsu N, et al. Assessment of timedependent changes in semen parameters in infertile men after microsurgical varicocelectomy. Urology. 2015; 86:48-51.

25. Marmar JL, Agarwal A, Prabakaran S, et al. Reassessing the value of varicocelectomy as a treatment for male subfertility with a new meta-analysis. Fertil Steril. 2007; 88:639-48.

26. Ariagno JI, Mendeluk GR, Furlan MJ, et al. Computer-aided sperm analysis: a useful tool to evaluate patient's response to varicocelectomy. Asian J Androl. 2017; 19:449-52.

27. Alargkof V, Kersten L, Stanislavov $R$, et al. Relationships between sperm DNA integrity and bulk semen parameters in Bulgarian patients with varicocele. Arch ItalUrol Androl. 2019; 91:125-129.

28. Zini A, Blumenfeld A, Libman J, Willis J. Beneficial effect of microsurgical varicocelectomy on human sperm DNA integrity. Hum Reprod. 2005; 20:1018-21.

29. Smit M, Romijn JC, Wildhagen MF, et al. Decreased sperm DNA fragmentation after surgical varicocelectomy is associated with increased pregnancy rate. J Urol. 2013; 189(1 Suppl):S146-50.

30. Inci K, Hascicek M, Kara O, et al. Sperm retrieval and intracytoplasmic sperm injection in men with nonobstructive azoospermia, and treated and untreated varicocele. J Urol. 2009; 182:1500-5.

31. Tournaye H. Male factor infertility and ART. Asian J Androl. 2012; 14:103-8.

32. Al Bakri A, Lo K, Grober E, et al. Time for improvement in semen parameters after varicocelectomy. J Urol. 2012; 187:227-31.

33. Nieschlag E, Hertle L, Fischedick A, et al. Update on treatment of varicocele: counselling as effective as occlusion of the vena spermatica. Hum Reprod. 1998; 13:2147-50.

\section{Correspondence}

Mohammad Ali Ghaed, MD - ghaed1982@gmail.com

Asaad Moradi, MD - Moradi.a@iums.ac.ir

Robab Maghsoudi, MD - rmaghsudy@yahoo.com

Urology Department, Firoozgar Hospital, Iran University

of Medical Sciences, Tehran (Iran)

Seyed Alireza Makkian, MD - alireza.makian@gmail.com Iran University of Medical Sciences, Tehran (Iran)

Alireza Gandomi-Mohammadabadi, MD (Corresponding Author) A.gandomi.researcher@gmail.com

Student Research Committee, School of Medicine, Iran University

of Medical Sciences, Tehran, Iran 